\title{
Le feedback structuré dans la formation postgraduée médicale: Mini-CEX et DOPS
}

\section{Berendonk, C. Beyeler, \\ R. Westkämper, M. Giger}

a Sont désignées comme médecinassistant, les personnes qui font une formation continue de médecin spécialiste.

b Sont désignés comme «formateur» les personnes responsable de la formation postgraduée des médecins-assistants: En règle générale, il s'agit de chefs de clinique, de responsables de services et de médecins chefs.

1 Epstein RM, Hundert EM. Defining and assessing professional competence. JAMA. 2002;287(2):226-35.

2 Holmboe ES, Hawkins RE. Methods for evaluating the clinical competence of residents in internal medicine: a review. Ann Intern Med. 1998;129(1):42-8.

3 Huddle TS, Heudebert GR. Taking apart the art: the risk of anatomiz ing clinical competence. Acad Med. 2007;82(6):536-41.

4 Dreyfus H, Dreyfus S. Mind over Machine. The Power of Human Intuition and Expertise in the Era of the Computer. New York: The Free Press; 1986.

Correspondance:

Dr C. Berendonk

Université de Berne

Institut de formation médicale

Unité des examens et de l'évaluation Inselspital 37a

CH-3010 Berne

christoph.berendonk@iml.unibe.ch

\section{Introduction}

Les objectifs de la formation médicale postgraduée sont définis comme suit dans la Loi sur les professions médicales (LPMéd Art 17): «La formation postgrade doit étendre et approfondir les connaissances, aptitudes, capacités, compétences sociales et comportements acquis lors de la formation universitaire de telle sorte que les personnes qui l'ont suivie soient à même d'exercer leur activité professionnelle sous leur propre responsabilité dans le domaine considéré.»

Ces objectifs sont contenus dans la définition d'Epstein qui résume la compétence médicale comme «la mise en œuvre habituelle et pondérée de la communication, du savoir, des aptitudes techniques, de l'argumentation clinique, des émotions, des valeurs et de la réflexion dans le travail concret tant pour le bien d'un individu que pour celui de tout un groupe de population» [1]. L'aptitude à accomplir une activité médicale découle de la maîtrise de compétences clé bien définies [2], mais résulte aussi d'un ensemble global qui ne peut se réduire à des aspects isolés et considérés individuellement [3].

La compétence médicale s'acquiert dans un processus continu qui s'étend sur la formation universitaire, la formation postgraduée et la formation continue. Le modèle par niveaux développé par les frères Dreyfus [4] décrit le développement de la compétence comme un processus qui évolue d'une attitude fondée sur des règles fixes vers une approche davantage orientée sur le contexte. Les personnes sans expériences vont se référer à des règles et les appliquer d'une manière schématique. Avec le temps, ces règles vont être utilisées avec des nuances et adaptées à chaque situation. Au fur et à mesure qu'il acquiert de l'expérience, l'apprenant prend conscience que le plus grand nombre possible de règles ne peut répondre à l'ensemble des situations qui se présentent à lui et qu'il doit prendre des décisions adaptées à la situation en faisant un choix et assumer par là même une responsabilité. Dans la mesure où le résultat dépend de ses choix, le praticien en formation se trouve ainsi impliqué au niveau de ses émotions. Ce processus évolue ensuite vers une compréhension toujours plus approfondie des principes, permettant au méde-

\section{Résumé}

Le médecin apprend son métier dans sa relation avec le patient et en collaboration avec lui lors de son travail clinique quotidien. La compétence médicale s'acquiert par la pratique systématique. La supervision du processus d'apprentissage et le feedback jouent dans ce processus un rôle central. L'évaluation formative permet de structurer ce processus d'apprentissage en fonction d'objectifs précis. Deux outils d'évaluation, le Mini-CEX (MiniClinical Evaluation Exercise) et la DOPS (Direct Observation of Procedural Skills), sont utilisés pour améliorer la formation postgraduée médicale. Le médecin-assistant ${ }^{a}$ est observé par un médecin formateur $^{\mathrm{b}}$ dans son travail au sein d'une clinique hospitalière et reçoit ensuite un feedback constructif de la part de ce dernier. L'observation directe, qui dure en règle générale un quart d'heure, se limite à une activité définie; ce processus est répété par d'autres formateurs lors de différentes situations cliniques sur un laps de temps déterminé de la formation postgraduée. Dans le cadre du projet pilote «Le feedback structuré dans la formation médicale postgraduée», l'applicabilité et l'acceptation du Mini-CEX/DOPS auprès des établissements de formation postgraduée en Suisse sont analysées pour la première fois. Les premières expériences réalisées révèlent les nombreuses possibilités d'utilisation de ces outils dans différentes spécialités. Les médecins-assistants émettent une appréciation positive du Mini-CEX/DOPS.

cin d'identifier les maladies classiques les plus courantes (pattern recognition) et de prendre des décisions sur la base d'une approche englobant de très nombreux aspects. Les experts peuvent également identifier les situations complexes avec peu d'informations explicites - et prendre des décisions adaptées au contexte. 
5 Ericsson KA. Deliberate practice and the acquisition and maintenance of expert performance in medicine and related domains. Acad Med 2004;79(10 Suppl): S70-81.

6 Schon D. Educating the reflective practitioner. San Francisco: Jossey-Bass; 1987.

7 Duffy FD, Holmboe ES. Selfassessment in lifelong learning and improving performance in practice: physician know thyself. JAMA. 2006;296(9):1137-9.

8 Norcini JJ, et al. The mini-CEX (clinical evaluation exercise): a preliminary investigation. Ann Intern Med. 1995;123(10):795-9.

9 Wragg A, Wade W, Fuller G Cowan G, Mills P. Assessing the performance of specialist registrars. Clin Med. 2003;3(2):131-4.

10 National Health Service. Modernising Medical Careers: Foundation Programmes. www.foundationprogramme.nhs uk/pages/home/training-andassessment. Dernierè visite: $1^{\mathrm{er}}$ avril 2008

11 Alves de Lima A, Henquin R, Thierer J, Paulin J, Lamari S, Belcastro F, van der Vleuten CP. A qualitative study of the impact on learning of the mini clinical evaluation exercise in postgraduate training. Med Teach. 2005;27(1):46-52.

12 Schuwirth LW, van der Vleuten CP. Assessment of medical competence in clinical education. Ned Tijdschr Geneeskd. 2005;149(49): 2752-5.

13 Hatala R, Ainslie M, Kassen BO, Mackie I, Roberts JM. Assessing the mini-Clinical Evaluation Exercise in comparison to a national specialty examination. Med Educ. 2006;40(10):950-6

14 Kogan JR, Hauer KE. Brief report: Use of the mini-clinical evaluation exercise in internal medicine core clerkships. J Gen Intern Med 2006;21(5):501-2.

15 Wilkinson JR, Crossley JG, Wragg A, Mills P, Cowan G, Wade W. Implementing workplace-based assessment across the medical specialties in the United Kingdom. Med Educ. 2008;42(4):364-73
Le développement de la compétence médicale n'est pas seulement une question d'expérience qui se mettrait en place automatiquement. Seule une pratique systématique et ciblée autorise le développement d'approches professionnelles élaborées permettant au médecin, dans sa pratique quotidienne, de planifier, d'agir et d'évaluer [5]. Ce n'est que par ce biais que le médecin va développer sa pratique et lui donner précision et efficacité. Le développement de sa réflexion par rapport aux expériences faites [6] et les réactions recueillies auprès des enseignants, des pairs ou des experts jouent un rôle significatif dans le développement et la consolidation de la compétence médicale [7].

En renforçant la supervision de l'activité clinique et le feedback structuré, les outils d'évaluation formative peuvent apporter un soutien précieux à la formation postgraduée.

\section{Outils d'évaluation formative}

\section{Le principe du Mini-CEX et du DOPS}

Le Mini-Clinical Evaluation Exercise (Mini-CEX) et le Direct Observation of Procedural Skills (DOPS) constituent deux procédures, qui, sur la base des réflexions ci-dessus, ont été conçues pour la formation postgraduée médicale. Le Mini-CEX met l'accent sur les aspects de la communication et de l'examen clinique, la DOPS privilégie les aptitudes manuelles et les interventions. Le Mini-CEX a été développé par l'American Board of Internal Medicine (USA) [8] et la DOPS par le Royal College of Physicians (UK) [9]. Depuis, ces deux outils sont utilisés dans de nombreux pays pour la formation préet postgraduée médicale [10-14].

Le Mini-CEX et la DOPS ont des priorités différentes, mais ils sont néanmoins identiques dans les principes qui les sous-tendent et dans leur structure. Les deux modèles sont en quelque sorte un instantané d'une interaction réelle entre le médecin et son patient et englobent les éléments suivants: 1 . observation directe par le formateur du médecin-assistant dans une situation clinique courante; 2 . évaluation de l'activité médicale selon une échelle comprenant sept critères; 3. feedback constructif du formateur; 4. élaboration conjointe d'un plan de mesures en fixant une procédure concrète pour éliminer les faiblesses constatées et renforcer les potentialités. L'observation dure environ quinze minutes; le feedback cinq minutes. Le Mini-CEX/ DOPS est répété à plusieurs reprises durant un certain laps de temps de la formation postgraduée. Des formateurs différents observent les
Renseignements sur les conflits d'intérêts possibles ou existants

Le Dr C. Berendonk, le Prof. C. Beyeler et le Dr R. Westkämper collaborent au sein de l'Unité des examens et de l'évaluation de l'Institut de formation médicale de l'Université de Berne. Le Dr M. Giger est président de la Commission pour la formation postgraduée et continue de la Fédération des médecins suisses (FMH). Le présent projet bénéficie d'un important soutien financier de la FMH.

médecins-assistants dans des situations diverses. L'objectif fixé est d'obtenir un feedback par rapport à différentes maladies de complexité diverse dans des lieux différents (lit hospitalier, service d'urgences, permanence, salle d'opération) et avec des priorités diverses (anamnèse, examen physique, évaluation et conseil, intervention). Ce n'est que dans ces conditions de diversité que le Mini-CEX/DOPS peut déployer tous ses effets au niveau de la formation et qu'il peut donner une image fidèle de la compétence médicale [15].

Pour répondre aux besoins des différentes disciplines médicales, les deux outils peuvent et doivent être modifiés pour tenir compte des contextes spécifiques.

\section{Les lieux de la mise en œuvre}

Dans le cadre d'un projet-pilote, le Mini-CEX est désormais utilisé au sein des départements de médecine interne de l'hôpital cantonal de Lucerne (depuis l'été 2006) et de l'hôpital cantonal des Grisons (depuis le printemps 2007) de même qu'au sein des institutions psychiatriques de Lucerne (emplacements de Lucerne et SaintUrban, depuis l'été 2007). Depuis l'automne 2007, la DOPS est également introduit parallèlement au Mini-CEX au sein de la clinique ORL de l'hôpital universitaire de Bâle. En préalable à leur implantation, une liste des critères portant sur les éléments à évaluer a été établie dans chacune des institutions concernées. Dans une deuxième phase, des collaborateurs de l'Institut de formation médicale ont introduit le personnel médical impliqué dans les principes de l'observation directe et du feedback. Compte tenu du fait que le Mini-CEX/DOPS a été conçu en priorité pour soutenir la formation postgraduée des médecins-assistants, l'initiative de la mise en œuvre des Mini-CEX/DOPS revenait par principe à eux.

\section{Premiers résultats}

Dans les quatre institutions concernées, jusqu'à février 2008, 124 Mini-CEX/DOPS ont été pratiqués auprès de 93 médecins-assistants par 37 formateurs. 


\section{Mise en œuvre pratique}

L'observation a duré en moyenne 16 minutes (valeur moyenne 15,8 , écart standard $\pm 4,1$ ) et le feedback a sollicité 5 bonnes minutes $(5,7 \pm 2,1)$; pour $40 \%$ des cas, le temps n'a pas été indiqué avec précision. 75 (61\%) Mini-CEX/DOPS ont été pratiqués dans un service hospitalier de médecine, $14(11 \%)$ au dispensaire, 10 (7\%) aux urgences, $8(6 \%)$ aux soins intensifs, $8(6 \%)$ en salle d'opération et 7 (6\%) dans la salle des ponctions (2 [2\%] sans indication). Quant à la complexité de la tâche à effectuer, 15 (12\%) parmi les formateurs l'ont jugée faible, 68 (55\%) comme moyenne et 14 (11\%) comme élevée (27 [22\%] n'ont pas répondu). Sur le total des 124 MiniCEX/DOPS, 15 (12\%) mettaient l'accent sur l'anamnèse, 19 (15\%) sur l'examen clinique, 25 (20\%) sur l'évaluation et le conseil, et 55 (45\%) sur une activité manuelle (10 [8\%] sans indication). A noter que les deux cliniques de médecine interne ne disposaient que du formulaire MiniCEX (et donc pas celui de la DOPS), et qu'elles présentent une proportion relativement élevée de $55 \%$ d'activités manuelles pour l'ensemble des Mini-CEX.

\section{Evaluation de la performance}

L'évaluation de l'impression générale et de dimensions spécifiques a été effectuée à partir d'une échelle de Likert de 9 points (1-3 en-deçà des attentes, 4-6 conforme aux attentes, 7-9 au-delà des attentes). L'évaluation de la performance a été faite aussi bien par les médecinsassistants eux-mêmes (autoévaluation) que par les formateurs (évaluation externe). Les médecinsassistants évaluent leur performance à 5,5 $\pm 1,1$ points, les formateurs à $6,3 \pm 1,1$. La corrélation entre l'autoévaluation des médecins-assistants et l'évaluation externe des formateurs s'est élevée à 0,58 . Les deux groupes ont donné la note la plus basse à l'examen clinique $(5,0 \pm 1,4$ médecins-assistants, $5,7 \pm 1,2$ formateurs), et la plus élevée à l'évaluation et conseil $(5,6 \pm 1,2$ médecins-assistants, 6,4 $\pm 1,3$ formateurs).

16 Veloski J, Boex JR, Grasberger MJ, Evans A, Wolfson DB. Systematic review of the literature on assessment, feedback and physicians' clinical performance: BEME Guide No. 7. Med Teach. 2006;28(2):117-28.

17 Jamtvedt G, Young JM, Kristoffersen DT, O'Brien MA, Oxman AD. Audit and feedback: effects on professional practice and health care outcomes. Cochrane Database Syst Rev. 2006;(2):CD000259. au contraire de la psychiatrie et du secteur ORL, où cette échelle correspondait au niveau de la formation postgraduée des médecins-assistants.

\section{Retour}

Il a été difficile de mettre en ouvre les quatre Mini-CEX/DOPS prévus par médecin-assistant et par année. A titre d'illustration de cette situation, nous citerons l'une des médecins cheffes impliquées dans le projet: «Nous avions convenu que la dette quérable était du ressort des médecins-assistants, ce qui les a fait hésiter. La mise en œuvre n'a pas posé de problèmes; le feedback est également apprécié. Néanmoins, en dépit de diverses sollicitations, l'ensemble des médecins-assistants n'ont pas régulièrement demandé à faire un Mini-CEX ou une DOPS ...».

\section{Satisfaction}

Malgré ces obstacles, les outils Mini-CEX/DOPS ont suscité un haut degré de satisfaction. Sur une échelle de Likert de 1-10 (1 = pas du tout, 10 = entièrement), les médecins-assistants l'ont évalué à 7,0 $\pm 1,8$ et les formateurs à $7,0 \pm 1,6$. A l'instar de l'évaluation de la performance, on note, en matière de satisfaction, des différences notables entre les cliniques. La satisfaction moyenne dans les cliniques varie chez les médecins-assistants de $6,0 \pm 1,6$ à $8,3 \pm 1,2$, elle est de $6,4 \pm 1,4$ à $8,1 \pm 1,1$ chez les formateurs.

\section{Discussion}

La compétence médicale s'acquiert dans la relation avec le patient et en collaboration avec lui dans le cadre de l'activité professionnelle clinique courante. Ce processus d'acquisition de compétences intègre aussi les erreurs. Pour parvenir à un degré élevé de compétence médicale, le feedback régulier d'experts est essentiel $[16,17]$. Les MiniCEX/DOPS sont des outils potentiellement aptes à améliorer la formation postgraduée médicale. D'une part, les MiniCEX/DOPS apportent au médecin-assistant un feedback constructif. D'autre part, ils permettent au formateur de se faire une idée précise de l'activité clinique des médecins-assistants. Le formateur peut ainsi encourager et soutenir les médecins-assistants avec pertinence.

Les évaluations des résultats de cette étude pilote montrent que le Mini-CEX/DOPS peut être intégré dans le quotidien hospitalier, qu'il couvre un large éventail des activités hospitalières, qu'il peut être utilisé dans des disciplines très diverses et qu'il bénéficie d'une large acceptation comme outil d'évaluation. Depuis le début 2008, l'introduction des outils Mini- 
18 Eva KW, Regehr G. Self-assessment in the health professions: a reformulation and research agenda. Acad Med. 2005; 80(10 Suppl):S46-54.

19 Holmboe ES, Yepes M, Williams F, Huot SJ. Feedback and the mini clinical evaluation exercise. J Gen Intern Med. 2004;19(5 Pt 2): 558-61.

20 Alves de Lima A, Barrero C, Baratta S, Castillo Costa Y, Bortman G, Carabajales J, et al. Validity, reliability, feasibility and satisfaction of the Mini-Clinical Evaluation Exercise (Mini-CEX) for cardiology residency training. Med Teach. 2007;29(8):785-90.
CEX/DOPS est activement préparée ou mise en œuvre dans des établissements de formation en chirurgie, gynécologie, obstétrique et pédiatrie.

Les raisons qui expliquent les réticences à mettre en œuvre le Mini-CEX/DOPS sont diverses. D'une part, il est compréhensible que toute innovation entraîne un surcroît de travail et peut être source d'insécurité, phénomènes qui doivent être pris au préalable en considération. D'autre part, le hiatus qui peut (éventuellement) apparaître dans le cadre d'un feedback régulier entre l'évaluation externe de sa compétence et sa propre évaluation voire une évaluation insuffisante de sa compétence par le responsable de la formation peut potentiellement entraîner une attitude d'évitement de la situation [18]. Il convient également de considérer le fait que la capacité à donner un feedback constructif est, pour tout formateur, un processus qui s'apprend et s'exerce [19]. Pour bien remplir sa fonction de formateur, un médecin cadre doit être d'abord un bon clinicien mais disposer aussi de compétences pédagogiques. Le Mini-CEX/DOPS ne peut déployer ses effets positifs sur la qualité de la formation que lorsque sa mise en œuvre répétée l'est par des formateurs différents. Sur la base de nos propres expériences et d'expériences réalisées ailleurs [20], cet objectif ne peut être visiblement atteint que lorsque des exigences, engageant les formateurs et les médecins-assistants, ont été formulées, les responsabilités ont été bien établies et que les ressources nécessaires ont été dégagées. Ces conditions peuvent être réglées dans le cadre d'un contrat de formation postgraduée et leur mise en œuvre être assurée par des formations ciblées.

Compte tenu des résultats positifs exposés ici mais aussi sur la base d'autres expériences, on pourra décider de l'introduction du Mini-CEX/ DOPS dans l'ensemble des établissements de formation postgraduée. Il s'agit désormais d'aborder avec toutes les instances impliquées la question de la responsabilité de la mise en œuvre, la formation des formateurs, l'administration, l'évaluation des données. Des questions fondamentales vont également se poser: quel doit être le niveau de qualité de la formation postgraduée en Suisse? Comment cette qualité doit-elle être atteinte, évaluée et attestée? Quelle place l'enseignement, donc la formation postgraduée, occupe-t-il dans les in-stitutions dispensant des prestations médicales à des patients?

\section{Remerciements}

Nous souhaitons remercier ici l'ensemble des médecins-assistants et formateurs participant au projet pilote dans les différentes cliniques impliquées pour leur participation. Nos remerciements s'adressent tout particulièrement au Prof. V. Briner et au Dr T. Hodel (Département de médecine interne, Hôpital cantonal de Lucerne), Prof. W. Reinhart, Dr T. Wieland et au Dr R. Strub (Département de médecine interne, Hôpital cantonal des Grisons), Dr J. Kurmann, Dr M. Kendall et Dr L. Schlegel (Psychiatrie Lucerne), Prof. M. Wolfensberger et au Dr A. Welge-Lüssen, privat-docent (Service ORL, Hôpital universitaire de Bâle) pour leur collaboration dans l'élaboration des listes de critères spécifiques aux disciplines médicales et le travail administratif réalisé sur les lieux de formation. Nos remerciements s'adressent également au Dr M. Perrig (Service de médecine interne générale, Hôpital de l'Ile, Berne) et au Dr S. Montagne (Institut de formation médicale, Université de Berne) pour leur soutien dans la mise sur pied d'ateliers, ainsi qu'au Dr C. Pfister (Institut de formation médicale, Université de Berne) pour les formations qu'il a donné dans d'autres cliniques pilotes. Enfin, nous remercions Madame A. Rogausch, Dr en sc. nat. (Institut de formation médicale, Université de Berne) pour la relecture critique du manuscrit et ses précieuses suggestions. 